\title{
Experience with a cholinesterase histochemical technique for rectal suction biopsies in the diagnosis of Hirschsprung's disease
}

\author{
P. H. TRIGG, R. BELIN, S. HABERKORN, W. J. LONG, H. H. NIXON, J. PLASCHKES, \\ L. SPITZ, AND G. H. WILLITAL
}

From the Hospital for Sick Children, Great Ormond Street, London

SYNOPSIS Cryostat sections from 160 rectal suction biopsies were stained for cholinesterases by the method of Karnovsky and Roots (1964) in an attempt to facilitate the diagnosis of Hirschsprung's disease.

The method proved at least as reliable as experienced assessment of paraffin haematoxylin-eosin sections, and appeared to offer the advantages of reduced scanning fatigue and superior demonstration of the increased cholinesterase-positive nerves in Hirschprung's disease.

Contrary to the findings of Meier-Ruge (1971) it was not possible to base a diagnosis on mucosal cholinesterase activity.

Rectal suction biopsies taken by the simple technique recently described by Noblett (1969) and Campbell and Noblett (1969) are surgically preferable to larger submucosal biopsies or to fullthickness techniques.

To the histopathologist, however, they may present problems of interpretation in the diagnosis of Hirschsprung's disease. This is in part due to the relatively diffuse nature of the superficial submucous plexus compared with the more familiar intermuscular plexus and to the rather smaller size of its ganglion cells and ganglion complexes.

We have applied a cholinesterase (ChE) histochemical technique to biopsies of this type in an attempt to make the submucosal nervous tissue more conspicuous and also to evaluate the significance of mucosal cholinesterase activity in the diagnosis of Hirschsprung's disease.

\section{Materials and Methods}

The biopsy site was in all cases estimated to be at least $1 \mathrm{~cm}$ above the proximal limit of the 'normal hypoganglionic zone' described by Aldridge and Campbell (1968).

One hundred and sixty biopsies were taken from 101 patients ranging in age from 2 days to 17 years,

Received for publication 23 October 1973. and were processed for cholinesterases. Whenever possible duplicate biopsies were also taken from an adjacent site, and were processed by a conventional histological method (fixation in neutral $10 \%$ formalin, embedding in paraffin wax, thin serial sections stained with haematoxylin and eosin). All the patients had presented with a clinical picture suggesting the possibility of Hirschsprung's disease, which was ultimately confirmed by definitive surgery in 33 of them and was excluded in the remainder.

HISTOCHEMICAL TECHNIQUE

The biopsy was immediately spread submucosadown onto a small block of animal spleen or liver (for ease of handling and cutting), and quick frozen in hexane cooled by dry ice in acetone. It was then cut at $15 \mu$ in a plane perpendicular to the flattened submucosal surface, as serial sections. The slides were air dried for a few minutes, immersed for 30 seconds in $10 \%$ neutral formalin at room temperature, and washed in distilled water. They were incubated for three hours at $37^{\circ} \mathrm{C}$ in the medium described by Karnovsky and Roots (1964), using an acetate buffer at $\mathrm{pH} 6.0$ and s-acetylthiocholine iodide (AThChI) as substrate. Inhibitors were not routinely used, so that the histochemical reaction presumably demonstrated both true (acetyl-) and pseudo-cholinesterase activity. After rinsing in 
distilled water the slides were counterstained in Ehrlich's haematoxylin to show the nuclei, differentiated in $1 \%$ acid alcohol, blued in running tap water, dehydrated, cleared in xylol and mounted in Canada balsam.

The specificity of the histochemical reaction was assessed by comparing serial sections of representative biopsies treated in different ways, including the use of selective acetylcholinesterase and pseudocholinesterase inhibitors. Counterstaining was omitted in these tests.

\section{Results}

APPEARANCE OF ChE PREPARATIONS

The end product of the histochemical reaction is a finely granular and highly refractile deposit of redbrown copper ferrocyanide. Localization in our sections was good.

\section{Submucous ganglia}

The submucous ganglia appear as strongly ChE-

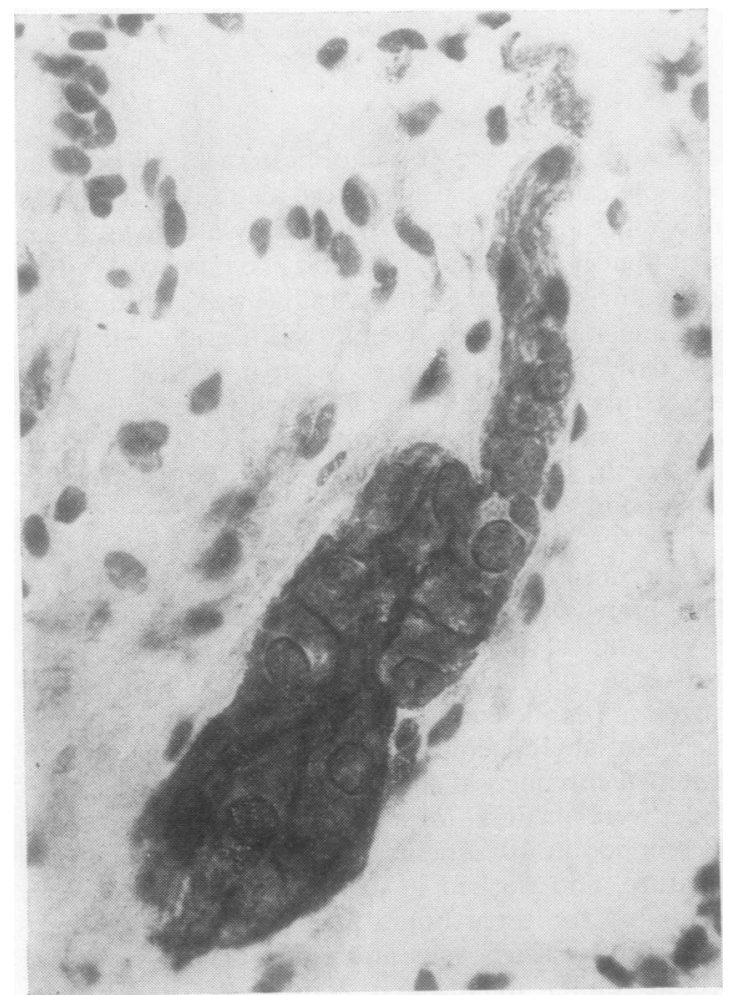

Fig 1

Fig 2

positive bodies of more or less clearly defined outline, though highly variable in size and shape The ganglion cells themselves are rather less ChE positive, and their loci typically appear as large, pale, approximately spherical spaces sharply differentiated from the surrounding matrix. Smaller and denser nuclei of supporting cells are generally visible within the ganglion (fig 1).

\section{Nerve bundles}

Nerve bundles are the only other strongly ChEpositive bodies present throughout the submucosa. In longitudinal section they appear as dense, more or less undulating ('zig-zag') bundles of fibres, in N $^{2}$ which individual axons are not distinguishable. $\omega$ Numerous elongated, densely basophilic nuclei of i supporting cells are seen along their length, especial- 공 ly around their periphery. Even in oblique or cross $ᄋ$ section their fibrous appearance and lack of ganglion cells distinguishes nerve bundles from ganglia (fig 2).

A loose mesh of fine ChE-positive nerves is also

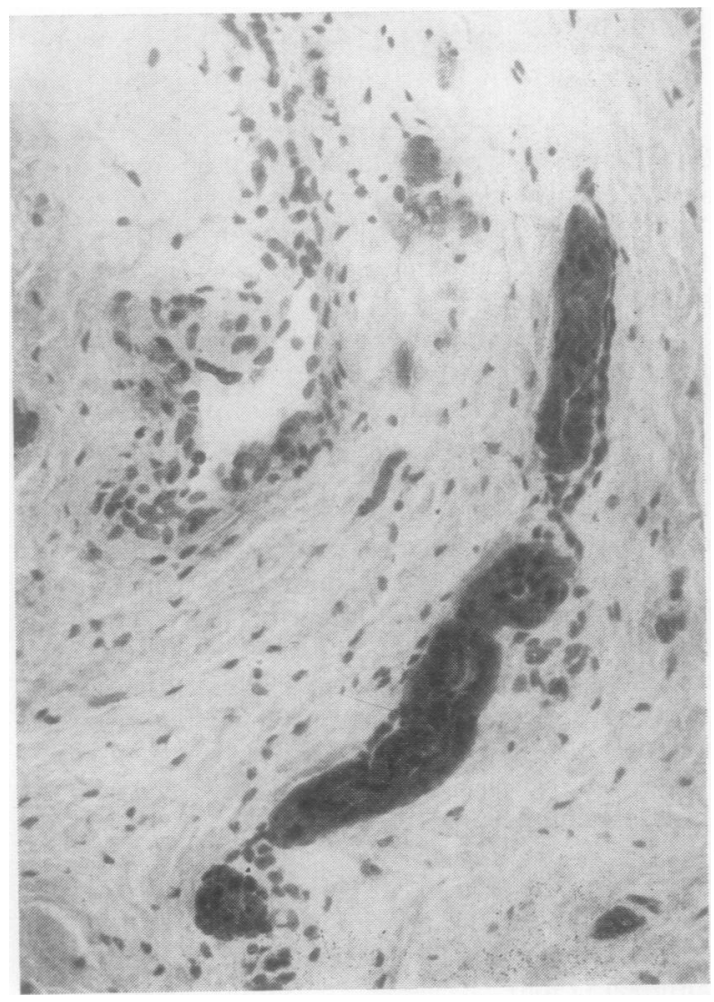

Fig 2 the characteristic 'halo' of paler cytoplasm around

Fig 1 A typical (fairly large) submucosal ganglion $(\times 375)$. Note the characteristic 'halo' of paler cytoplasm around each ganglion cell nucleus.

Fig 2 A typical (fairly large) ChE-positive submucosal nerve (from a ganglionic biopsy) $(\times 150)$. 


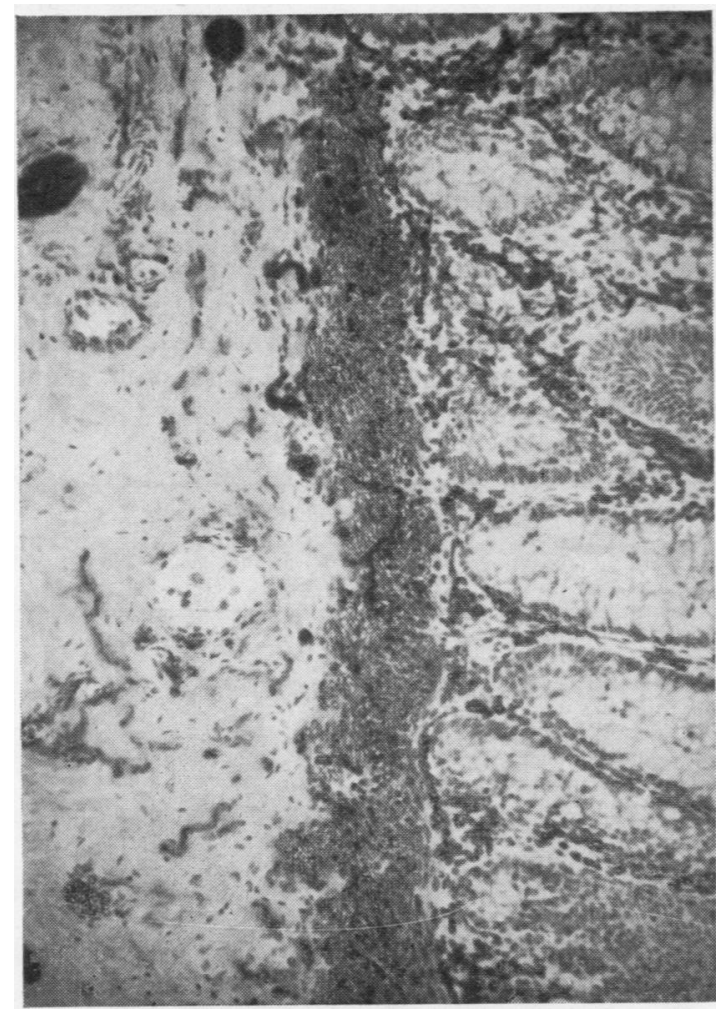

Fig 3 Muscularis mucosae from a Hirschsprung biopsy ( $\times$ 190). The superabundant fine nerves are clearly visible against the paler ChE coloration of the smooth muscle, and they are commonly seen even in those Hirschsprung biopsies which do not contain abnormally large or numerous submucosal nerve trunks.

generally visible around the submucosal blood vessels.

\section{Muscularis mucosa}

The muscularis mucosae appears as a fairly dense ChE-positive network surrounding the blue-stained elongated muscle-cell nuclei. Examination under high power suggests that the ChE activity is concentrated around the periphery of the muscle cells (fig 3).

Mucosal cholinesterase activity is in most biopsies due in large part to an anastomosing network of fine fibres in the lamina propria, running more or less parallel with the crypts. Their appearance suggests that they are small bundles of nerve fibres. Both their apparent number and their density of histochemical coloration contribute to the overall variability of $\mathrm{ChE}$ activity.

In addition, variable patches of intensely ChEpositive material of a more disorganized fibrous appearance, associated with brown-staining oval cell nuclei, occur in the lamina propria of many biopsies, both ganglionic and Hirschsprung's. These fibres do not appear to be nerves: in some biopsies they occur throughout the mucosa (with variable breakdown of the normal crypt architecture) and constitute the dominant contribution to $\mathrm{ChE}$ activity. They are consistently seen in biopsies which appear congested on macroscopic examination. They probably consist of extravasated plasma proteins and blood cells, and may in some cases be due to the biopsy procedure itself. Similar patches are sometimes also seen in the submucosa.

As well as a brown coloration of the distal cytoplasm of the epithelial cells bordering the lumen, a thin line of ChE-activity is sometimes seen just deep to the basement membrane of the epithelium bordering the lumen. Finally, small branches of the muscularis mucosae penetrate the lamina propria and contribute to the overall activity observed.

\section{ASSESSMENT OF MUCOSAL CHOLINESTERASE ACTIVITY}

Assessment of mucosal cholinesterase activity presented several problems. As described above, the pattern as well as the overall average density of ChE coloration varied in several ways. For the purposes of numerical analysis, however, the only practicable criterion was found to be the overall density of the histochemical deposit, regardless of fine histological appearance. The strongly ChEpositive cores of lymph follicles, which were commonly seen in biopsies of both types, were, however, excluded from this assessment.

The biopsies were subjectively ascribed to one of five arbitrary categories thus:

\section{Mucosal activity light}

(for the most part barely visible, with at most sporadic patches of higher density) .. rating 1

\section{Mucosal activity moderate}

$\begin{array}{llllll}\text { Mostly patchy } & \text {. } & \text {. } & \text {. } & \text {. } & \text { rating } 2\end{array}$

Mostly diffuse $\quad$. $\quad \ldots \quad \ldots \quad \ldots \quad$ rating 3

\section{Mucosal activity intense}

(strikingly increased throughout the greater area of the mucosa)

$\begin{array}{llllll}\text { Mostly patchy } & \text {. } & \text {. } & \text {.. } & \text {. } & \text { rating } 4\end{array}$ Mostly diffuse $\quad \ldots \quad \ldots \quad \ldots \quad \ldots \quad \ldots$ rating 5

A higher rating figure infers a higher overall average density of coloration.

\section{Numerical Data}

\section{ADEQUATE BIOPSIES}

Table I shows the distribution of ganglia in biopsies 


\begin{tabular}{lll}
\hline $\begin{array}{l}\text { Distribution of Ganglia and } \\
\text { Submucosal Nerves }\end{array}$ & \multicolumn{2}{l}{ Definitive Diagnosis } \\
\cline { 2 - 3 } & $\begin{array}{l}\text { Hirschsprung's } \\
\text { Disease }\end{array}$ & $\begin{array}{l}\text { Other } \\
\text { Disorders }\end{array}$ \\
\hline Ganglia identified & & \\
Few nerves & 0 & 41 \\
Moderate nerves & 0 & 6 \\
Many nerves & 0 & 0 \\
Total & 0 & 47 \\
Ganglia not identified & & \\
Few nerves & 0 & 8 \\
Moderate nerves & 4 & 1 \\
Many nerves & 31 & 0 \\
Total & 35 & 9 \\
Total adequate biopsies & & 91 \\
\hline
\end{tabular}

Table I Ganglion and submucosal nerve distribution in biopsies with adequate quantity and quality of submucosa

\begin{tabular}{lll}
\hline & \multicolumn{2}{c}{ Definitive Diagnosis } \\
\cline { 2 - 3 } & $\begin{array}{ll}\text { Hirschsprung's } \\
\text { Disease }\end{array}$ & $\begin{array}{l}\text { Other } \\
\text { Disorders }\end{array}$ \\
\hline Ganglia identified & 0 & 24 \\
Ganglia not identified & $22^{1}$ & 23 \\
Total inadequate biopsies & & 69
\end{tabular}

Table II Ganglion distribution in biopsies judged inadequate

${ }^{1}$ Only 14 of these biopsies permitted assessment of submucosal nerves. In all of these the number of nerves was much increased ('many').

\begin{tabular}{|c|c|c|c|c|}
\hline \multicolumn{2}{|c|}{ Mucosal ChE Activity } & \multirow[t]{2}{*}{ Rating } & \multicolumn{2}{|c|}{ Definitive Diagnosis } \\
\hline & & & $\begin{array}{l}\text { Hirschsprung's } \\
\text { Disease }\end{array}$ & $\begin{array}{l}\text { Other } \\
\text { Disorders }\end{array}$ \\
\hline Light & & 1 & 1 & 27 \\
\hline \multirow{2}{*}{ Moderate } & Patchy & 2 & 3 & 39 \\
\hline & Diffuse & 3 & 19 & 19 \\
\hline \multirow[t]{2}{*}{ Intense } & Patchy & 4 & 3 & 7 \\
\hline & Diffuse & 5 & 28 & 1 \\
\hline \multicolumn{3}{|c|}{ Total biopsies assessed } & 54 & 93 \\
\hline
\end{tabular}

Table III Mucosal cholinesterase activity of biopsies compared with definitive diagnosis

judged adequate in terms of the amount of submucosa of acceptable histological quality available for inspection (as in tables II and III, numbers refer to biopsies and not to patients). The distribution of ChE-positive nerves in the submucosa is also indicated, assessed subjectively as 'few', 'moderate', or 'many', a combined estimate of number and size.

INADEQUATE BIOPSIES

Table II shows the ganglion distribution in biopsies judged inadequate on the above basis and also includes three biopsies in which there was histological evidence that the biopsy site was too low, close to the mucocutaneous junction.
MUCOSAL CHOLINESTERASE

Table III shows the mucosal cholinesterase rating $\overrightarrow{\vec{F}}$ of the biopsies against definitive diagnosis. Thirteen $\stackrel{9}{\rightarrow}$ biopsies had to be excluded as unsuitable for this assessment.

As an indication of the reproducibility of estimates

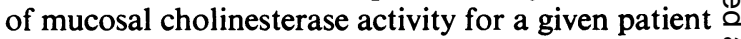
and height of biopsy, we compared mucosal ratings of pairs of biopsies, each pair taken from one patient $\overrightarrow{0}$ within an estimated $1 \mathrm{~cm}$ height from each other. In only four instances out of 66 did the pairs differ by $\vec{\omega}$ more than one rating.

\begin{tabular}{lll}
\hline Biopsy Findings & \multicolumn{2}{l}{ Definitive Diagnosis } \\
\cline { 2 - 3 } & $\begin{array}{l}\text { Hirschsprung's } \\
\text { Disease }\end{array}$ & $\begin{array}{l}\text { Other } \\
\text { Disorders }\end{array}$ \\
\hline $\begin{array}{c}\text { Hirschsprung's disease confirmed } \\
\text { (no ganglia seen, many } \\
\text { submucosal nerves) }\end{array}$ & 19 & 0 \\
$\begin{array}{c}\text { Hirschsprung's disease suggested } \\
\text { (no ganglia seen, moderate no. } \\
\text { of submucosal nerves) }\end{array}$ & 4 & 0 \\
$\begin{array}{c}\text { Bowel ganglionic (ganglia } \\
\text { positively identified) }\end{array}$ & 0 & 40 \\
$\begin{array}{c}\text { Bowel probably ganglionic } \\
\text { (ganglia tentatively identified) } \\
\text { Equivocal (no ganglia seen, few } \\
\text { submucosal nerves) }\end{array}$ & 0 & 1 \\
$\begin{array}{c}\text { Total patients with adequate } \\
\text { biopsies }\end{array}$ & 0 & 3 \\
\hline
\end{tabular}

Table IV Findings from ChE biopsies of 67 patients with an adequate biopsy

In an attempt to ascertain whether factors other then Hirschsprung's disease influenced mucosal ChE activity, the mucosal rating was plotted against age and also against the incidence of the enterocolitis which sometimes complicates the disease. In neither case could a relationship be established. Nor could any correlation be determined within the Hirschsprung biopsies between the mucosal ChE rating and the length of the aganglionic segment.

\section{Specificity Tests}

Preincubation of the slides in a solution of N-ethyl $N$ maleimide (at $0 \cdot 1 \mathrm{M}$ in a $0 \cdot 1 \mathrm{M}$ phosphate buffer, $\omega$ $\mathrm{pH} 7.4$, at $37^{\circ} \mathrm{C}$ for 15 minutes) resulted in some reduction in nuclear staining, especially noticeables in the mucosa. Karnovsky and Roots (1964)ळ suggest the use of this reagent for abolishing possible ${ }^{+}$ reduction of the potassium ferricyanide by sulphy- $\frac{T}{T}$ dryl groups). The staining reactions of other tissue $\frac{\mathbb{\mathbb { D }}}{\mathrm{O}}$ components were apparently unaltered.

Inclusion of the selective pseudocholinesterase $\mathbb{\mathbb { Q }}$ inhibitor iso-OMPA in the incubation medium ato $7 \times 10^{-5} \mathrm{M}$ resulted in a reduction in staining 
intensity in the muscularis mucosae and to a lesser extent in the ganglia. Other staining reactions were unaltered.

Inclusion of the selective acetylcholinesterase inhibitor BW $284 C 51$ in the incubation medium at $3 \times 10^{-5} \mathrm{M}$ resulted in abolition of coloration except in the muscularis mucosae and in the ganglia, in both of which the staining intensity was greatly reduced.

Inclusion of both iso-OMPA and BW 284C51 at these concentrations abolished all but the faintest coloration in the muscularis mucosae.

\section{Discussion}

The interpretation of rectal suction biopsies in the diagnosis of Hirschsprung's disease presents several critical questions to the histopathologist. The biopsy site must be selected above the 'normal hypoganglionic zone' described by Aldridge and Campbell (1968), but must be low enough to avoid missing an 'ultrashort segment' variant of the disease. Moreover, since the biopsy is performed without proctoscopy, and on an unsedated child, and in view of the elasticity of the rectal mucosa and the probability of encountering rectal folds, care is necessary to ensure that the surgeon's estimate of biopsy height is reliable. The apparatus must be capable of providing undamaged biopsies of adequate size and depth, and the biopsy must be orientated and processed with special care because of its small size.

Gherardi (1960) and others have established that the limit of aganglionosis in Hirschsprung's disease coincides closely in the submucosal and intermuscular plexuses. Aldridge and Campbell (1968) have significantly advanced our knowledge of the distribution of submucosal ganglia in the normal rectum at different ages, so that the optimum biopsy level for a child of any given age may be estimated with some confidence. Yet many pathologists are still reluctant to make a positive diagnosis of Hirschsprung's disease on the basis of rectal suction biopsies, possibly due both to doubt as to the amount of submucosa that must be scanned before absence of ganglion cells is indicative of aganglionosis at that level; and the relative difficulty and tedium of accurate identification and assessment of submucosal ganglia and nerves by comparison with the more compact and familiar intermuscular plexus.

Several workers have introduced histochemical methods in an attempt to facilitate the interpretation of these biopsies. Gannon, Burnstock, Noblett, and Campbell (1969) have applied the formaldehydeinduced fluorescence technique for catecholamines developed by Falck and Owman (1965). They were able to diagnose all of eight cases of Hirschsprung's disease studied in this way 'by looking at one, or at most several, sections', on the basis of a massive increase in the fluorescent nerves in the submucosa and muscularis mucosae, compared with normal controls. Though they assert that this method serves as a 'useful corollary to conventional histological methods', the technique is demanding in time and apparatus and is notoriously capricious in its working. Furthermore, Garrett and Howard (1969) found in a similar series at least one apparent exception to these observations.

Garrett and Howard (1969) and Lake (1972) have shown the practicability of rapid non-specific esterase techniques in the demonstration of nerves and ganglia in intraoperative Hirschsprung biopsies. We have applied such a technique to rectal suction biopsies, but found the cholinesterase technique described here more revealing and, apart from the speed, no less convenient.

Meier-Ruge (1968), Moser, Widmer, and MeierRuge (1971) and Meier-Ruge, Lutterbeck, Herzog, Morger, Moser, and Schärli (1972) report on the application of acetylcholinesterase techniques to rectal suction biopsies. Meier-Ruge (1971) and MeierRuge, Bielser, Wiederhold, and Meyerhofer (1971) have routinely used the method of Karnovsky and Roots, as slightly modified by el-Badawi and Schenk (1967), as well as Gomori's (1952) modification of the method of Koelle and Friedenwald. Drawing from experience with a large series of some 214 biopsies from as many patients, he states that a positive diagnosis can be made from rectal suction biopsies consisting of mucosa alone, based on a massive increase in AChE activity in the lamina propria, which he finds to be proportional to the severity of clinical presentation. In normal rectal mucosa, he finds, by contrast, 'virtually imperceptible' mucosal AChE activity (with 90 minutes' incubation). Furthermore he asserts that mucosal AChE activity affords precise diagnosis even 'from biopsies of the anal mucosa and within the first $3 \mathrm{~cm}$ of the rectum proximal to the anal ring'.

Garrett, Howard, and Nixon (1969a, 1969b), in 19 cases of Hirschsprung's disease examined, established a positive correlation between the severity of clinical presentation and numbers of AChE-positive nerves in the circular muscle of the distal aganglionic bowel. Of these 19, three cases which failed to show a classic constricted segment on barium enema had even fewer AChE-positive nerves in the circular muscle than did normal controls, and, contrary to Meier-Ruge's findings, also had few in the mucosa. In these three patients presentation was mild (constipation).

We found that from a technical point of view our method presented no special problems, and that 
serial sections could with practice be cut on the cryostat with ease and speed, mounting on average some 24 sections per slide. Ideally, a biopsy could be ready for examination within some four hours of receipt. In the routine pathology laboratory, however, geared primarily to paraffin sections stained with haematoxylin and eosin, considerable extra labour would probably be required to produce cholinesterase sections solely for rectal suction biopsies.

The histochemical reaction was generally consistent enough to permit a meaningful subjective comparison of the ChE activity of different biopsies. The animal tissue on which the biopsies were mounted provided a useful control in this respect. A few biopsies failed to stain properly, ChE-positive material appearing a dirty green colour: this was traced to contamination by excess mineral oil used to lubricate the biopsy forceps. A distinct but slight increase in the overall histochemical activity was noted when one sample of AThChI was replaced by a fresher sample from a different source, and allowance was made for this in the assessment. Cold storage of the biopsies (protected against drying) or the air drying of slides at $-30^{\circ} \mathrm{C}$ did not appear to diminish histochemical activity over a period of a few days.

The relatively thick sections and protracted incubation period were chosen to demonstrate mucosal ChE activity to best advantage at the expense of some loss of cytological detail. Identification of ganglia was accordingly more dependent on their overall configuration (though highly distinctive using this method), rather than on the fine cytological characterization of ganglion cells, particularly of the nuclear detail, that fine paraffin sections permit.

Of the 91 biopsies judged 'adequate' (table I) Hirschsprung's disease could be excluded by the presence of ganglia in $47(52 \%)$, and was strongly suggested in $31(34 \%)$ by the absence of ganglia coupled with a marked increase in the number and/or size of ChE-positive nerves in the submucosa. The remaining $13(14 \%)$ adequate biopsies were not diagnostic. In no case was the disease wrongly excluded or confirmed.

By comparison, of the 69 biopsies judged 'inadequate' from a priori considerations (table II), 24 (35\%) excluded Hirschsprung's disease and 14 $(20 \%)$ tended to confirm it, leaving 31 (45\%) equivocal. Again no errors of diagnosis were made.

If the rate of diagnosis is considered per patient rather than per biopsy we find that of 67 patients from whom an adequate biopsy was obtained, a correct diagnosis was made for $59(88 \%)$, and diagnosis was suggested (though less definitely) in a further five patients $(7 \%)$.

We found that both ChE-positive nerves and $\stackrel{5}{\frac{9}{8}}$ ganglia in the submucosa could be scanned rapidly $\frac{?}{\circ}$ and accurately, using low power $(x 100)$ with only $\frac{\sigma}{\bar{c}}$. occasional need for a higher power. The conspicuous- $\frac{\vec{\sigma}}{\widetilde{D}}$ ness of nervous tissue against the submucosal ground $\varrho$ matter, in our opinion, made scanning of serial \& sections for ganglia less tiring than with conven- $\overrightarrow{0}$ tional haematoxylin and eosin preparations. In particular we felt that the superabundant fine nerves $\vec{\omega}$ in and near the muscularis mucosae, which were $\frac{\Omega}{\circ}$ demonstrable in nearly all Hirschsprung biopsies, were easier to assess. But such comparisons are $N$ necessarily subjective, and for the pathologist who $\omega$ is familiar with the interpretation of paraffin/ haematoxylin and eosin sections of these biopsies, $O$ the advantages may be offset by the problems of $工$ adjustment to a radically different type of preparation.

A visiting pathologist with special interest and $\stackrel{\widehat{\partial}}{\supset}$ experience in this field examined a duplicate set of $\vec{\oplus}$ serial paraffin/haematoxylin and eosin preparations of biopsies of the last 62 of the series presented here: his diagnostic rate almost exactly coincided with our own (Carter, 1972).

Thus, apart from any advantages of convenience, any ultimate advantage of this method must lie with the diagnostic potential of the mucosal cholines- $\varrho$ terase activity. But though we found (table III) that $\overrightarrow{\overrightarrow{0}}$ most $(71 \%)$ non-Hirschsprung biopsies had a low 3 mucosal rating (1 or 2$)$ and most $(93 \%)$ Hirschsprung biopsies had a higher rating $(3,4$, or 5$)$ there was a considerable overlapping group, so that in our hands the mucosal cholinesterase activity is not alone a reliable diagnostic criterion.

In this respect our results differ markedly from those of Meier-Ruge and his colleagues (1972), who found high mucosal AChE activity to be a $100 \%$ o reliable criterion for the diagnosis of Hirschsprung's disease in a series of 214 patients. This could con- $\frac{D}{0}$ ceivably be due to their routine use of OMPA as a pseudocholinesterase inhibitor, although our speci- N ficity tests suggest that its use would not have $N$ significantly affected our mucosal observations. N Alternatively the discrepancy may be due to other ${ }^{\omega}$ differences in technique, including perhaps the biopsy procedure itself: or differences in inter- $\stackrel{0}{0}$ pretation may be involved, our rating being based $\stackrel{\oplus}{\Phi}$ solely on the density of histochemical coloration without interpretation of its origin as nervous, 'specific', or otherwise.

Finally it is noteworthy that one biopsy from this $\frac{\widehat{Q}}{\mathbb{Q}}$ series, taken in the routine fashion from a premature $\stackrel{0}{0}$ neonate, contained in addition both circular and longitudinal muscle (and a ganglionic myenteric 
plexus). The biopsy was only of average (absolute) depth but the bowel wall was unusually thin. A similar biopsy has since been taken from a premature baby (not of this series). No complications ensued in either case, but the bowel was presumably nearly perforated, suggesting that a modified technique might be advisable for premature babies. Furthermore, Carter (1972) remarked on the difficulty of identifying ganglion cells in the submucosa of haematoxylin and eosin preparations of biopsies from premature infants. In ChE preparations the ganglia tend to be atypical, with a light histochemical deposit and closely packed ganglion cell nuclei: their identification, however, is unambiguous (fig 4).

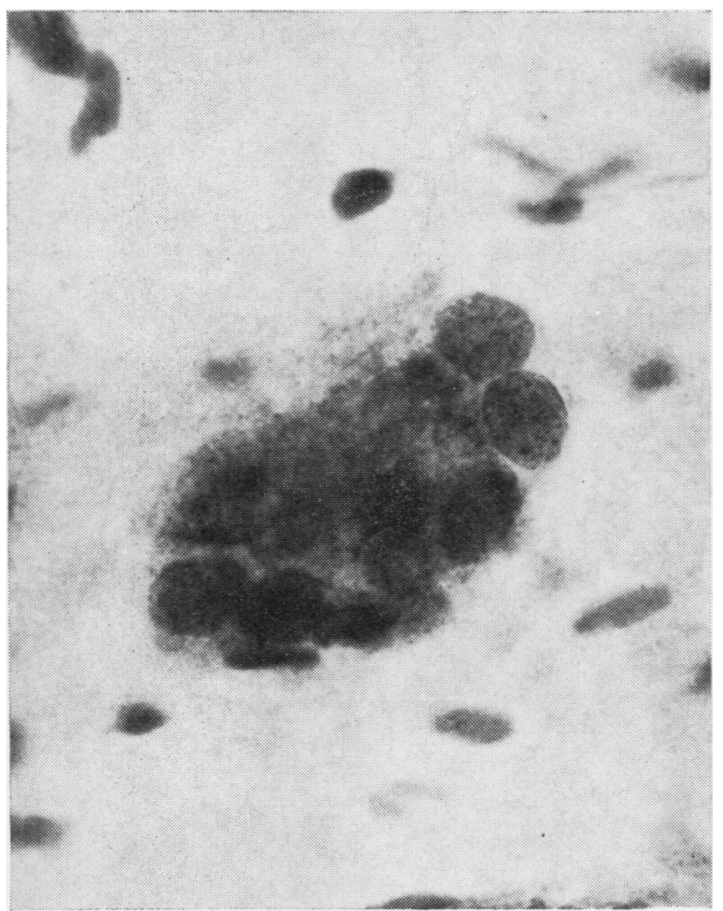

Fig 4 Submucosal ganglion from a premature infant. $(\times 450)$.
P. H. T. was in receipt of a grant from Reckett and Colman. W. J. L. was supported by the M.R.C. L. S. held a Smith and Nephew fellowship.

\section{References}

Aldridge, R. T., and Campbell, P. E. (1968). Ganglion cell distribution in the normal rectum and anal canal: a basis for the diagnosis of Hirschsprung's disease by anorectal biopsy. J. pediat. Surg., $3,475-490$.

Campbell, P. E., and Noblett, H. R. (1969). Experience with rectal suction biopsy in the diagnosis of Hirschsprung's disease. J. pediat. Surg., 4, 410-415.

Carter, R. F. (1972). Personal communication.

El-Badawi, A., and Schenk, E. A. (1967). Histochemical methods for separate, consecutive, and simultaneous demonstration of acetylcholinesterase and norepinephrine in cryostat sections. J. Histochem. Cytochem., 15, 580-588.

Falck, B., and Owman, C. (1965). A detailed methodological description of the fluorescence method for the cellular demonstration of biogenic monoamines. Acta Univ. lund., Sect. II, no. 7.

Gannon, B. J., Burnstock, G., Noblett, H. R., and Campbell, P. E. (1969). Histochemical diagnosis of Hirschsprung's disease. (Letter). Lancet, 1, 894.

Garrett, J. R., and Howard, E. R. (1969). Histochemistry and the pathology of Hirschsprung's disease. Proc. roy. microsc. Soc., 4, 76-78.

Garrett, J. R., Howard, E. R., and Nixon, H. H. (1969a). Autonomic nerves in rectum and colon in Hirschsprung's disease. Arch. Dis. Childh., 44, 406-417.

Garrett, J. R., Howard, E. R., and Nixon, H. H. (1969b). Histochemical diagnosis of Hirschsprung's disease. (Letter). Lancet, 2, 436.

Gherardi, G. J. (1960). Pathology of the ganglionic-aganglionic junction in congenital megacolon. Arch. Path., 69, 520-523.

Gomori, G. (1952). Microscopic Histochemistry: Principles and Practice. The University of Chicago Press, Chicago.

Karnovsky, M. J., and Roots, L. (1964). A 'direct-colouring' thiocholine method for cholinesterases. (Letter). J. Histochem. Cytochem., 12, 219-221.

Lake, B. D. (1972). Personal communication.

Meier-Ruge, W. (1968). Das Megacolon. Seine Diagnose und Pathophysiologie. Virchows Arch. path. Anat., 344, 67-85.

Meier-Ruge, W. (1971). Personal communication.

Meier-Ruge, W., Bielser, W. Jr., Wiederhold, K. H., and Meyenhofer, M. (1971). Incubation media for routine laboratory work on enzyme histotopochemistry. Beitr. path. Anat., 144, 409-431.

Meier-Ruge, W., Lutterbeck, P. M., Herzog, B., Morger, R., Moser, R., and Schärli, A. (1972). Acetylcholinesterase activity in suction biopsies of the rectum in the diagnosis of Hirschsprung's disease. J. pediat. Surg., 7, 11-17.

Moser, R., Widmer, A., and Meier-Ruge, W. (1971). Zur enzymhistochemischen Diagnostik des Morbus Hirschsprung. Schweiz. med. Wschr., 101, 109-115.

Noblett, H. R. (1969). A rectal suction biopsy tube for use in the diagnosis of Hirschsprung's disease. J. pediat. Surg., 4, 406-409. 